The Journal of Geometric Analysis

Volume 7. Number 3, 1997

\title{
Correction to: \\ Quadratic Presentations and Nilpotent Kähler Groups
}

\author{
By James A. Carlson and Domingo Toledo
}

First correction. It has been pointed out to us by S. Chen that the inductive step (2.3) in the construction of 1-formal, 1-minimal models is not correct. By 1-formal, 1-minimal model we mean what is called formal 1-minimal model in the first paragraph of Section 2. We proceed to describe a correct inductive step.

Let $V_{1}$ denote the vector space $V$ of Section 2 over the field $k$ (one of $\mathbb{Q}, \mathbb{R}$ or $\mathbb{C}$ ). Let $V_{2}$ denote the space $C_{0}$ defined by (2.1), namely the kernel of the cup-product. Assume that $i \geq 2$, that $V_{1}, \ldots, V_{i}$ have been defined, let $V^{i}=V_{1} \oplus \cdots \oplus V_{i}$, and let $\mathcal{M}_{i}=\Lambda\left(V^{i}\right)$. Assume that the differential $d: \mathcal{M}_{i} \rightarrow \mathcal{M}_{i}$ has also been defined and that it commutes with the action of the multiplicative group $k^{*}$ on $\Lambda\left(V^{i}\right)$ by the algebra automorphisms defined by letting $\lambda \in k^{*}$ act on $V_{j}$ as multiplication by $\lambda^{j}$. If $W$ is a vector space with a $k^{*}$ action by semisimple automorphisms, let $W(j)$ denote the vector subspace where $\lambda$ acts by multiplication by $\lambda^{j}$. Then define

$$
V_{i+1}=\Lambda^{2}\left(V^{i}\right)(i+1) \cap \operatorname{ker}(d),
$$

$\mathcal{M}_{i+1}=\Lambda\left(V^{i+1}\right)$, and the differential $d$ on $\mathcal{M}_{i+1}$ to be the Hirsch extension of the differential algebra $\mathcal{M}_{i}$ obtained by defining $d \mid V_{i+1}$ to be the inclusion of $V_{i+1}$ in $\Lambda^{2}\left(V^{i}\right)$.

This definition is clearly correct because, by the inductive assumption of a $k^{*}$ action, we have a decomposition

$$
H^{2}\left(\mathcal{M}_{i}\right)=\oplus_{j=2}^{2 i} H^{2}\left(\mathcal{M}_{i}\right)(j)
$$

We are inductively arranging $H^{2}\left(\mathcal{M}_{i}\right)(j)=0$ for $2<j \leq i$ to achieve in the limit that $H^{2}(\mathcal{M})(j)=0$ for $j \neq 2$, which is equivalent to 1-formality. Now $V_{i+1}$ is isomorphic to $H^{2}\left(\mathcal{M}_{i}\right)(i+1)$, and $d$ is defined precisely to then make $H^{2}\left(\mathcal{M}_{i+1}\right)(j)=0$ for $2<j \leq i+1$.

The reason we choose this procedure is that the $k^{*}$ action is easily defined. Another correct procedure, closer to the definition of 1-formality, would have been to have a $k^{*}$ action on $V_{i}$ with more weights, and to define $V_{i+1}$ to be $\oplus_{j>2} H^{2}\left(\mathcal{M}_{i}\right)(j)$. 
With our chosen procedure, $\mathcal{M}_{i}$ is 1 -formal if and only if $H^{2}\left(\mathcal{M}_{i}\right)(j)=0$ for all $j>2$. Note that this is in general a stronger condition than the vanishing of $V_{i+1}$.

The relation of the chosen procedure to Section 2 and the error there is now easily explained. The space $C_{i}$ defined by (2.3) is the subspace $V_{i+2} \cap\left(V_{1} \otimes V_{i+1}\right)$ of

$$
\Lambda^{2}\left(V^{i+1}\right)=V_{1} \otimes V_{1+1} \oplus V_{2} \otimes V_{1} \oplus \cdots
$$

which in general is a proper subspace of $V_{i+2}$.

Fortunately, this error leaves all but one assertion in the paper unaffected. The reason is that for small $i$ there is little difference between the correct and incorrect procedures. Namely, we have the following assertions:

Lemma 0.1. For any $V_{1}, V_{2}$ and injective $d: V_{2} \rightarrow \Lambda^{2} V_{1}$, if $V_{i}$ and $\mathcal{M}_{i}$ are defined inductively as above, then

l. $H^{2}\left(\mathcal{M}_{2}\right)(4)=0$.

2. $H^{2}\left(\mathcal{M}_{3}\right)(5)=H^{2}\left(\mathcal{M}_{3}\right)(6)=0$.

3. $H^{2}\left(\mathcal{M}_{4}\right)(7)=H^{2}\left(\mathcal{M}_{4}\right)(8)=0$.

4. $V_{3}=C_{1}$, where $C_{1}$ is defined in (2.2).

5. If $\operatorname{dim}\left(V_{3}\right)=1$ and $d\left(V_{2}\right)$ contains no decomposable element of $\Lambda^{2} V_{1}$, then $V_{4} \cong C_{2}$, where $C_{2}$ is defined as in (2.3). In fact, under these assumptions $V_{4}=0$ unless $V_{3} \subset V_{1} \otimes V_{2}$ consists of decomposable elements, in which case $\operatorname{dim}\left(V_{4}\right)=\operatorname{dim}\left(C_{2}\right)=1$.

Proof. We prove typical assertions 2 and 5. To prove 2, note that

$$
H^{2}\left(\mathcal{M}_{3}\right)(5)=\operatorname{ker}\left(V_{2} \otimes V_{3} \rightarrow V_{1} \otimes \Lambda^{2} V_{2} \oplus \Lambda^{2} V_{1} \otimes V_{3}\right)
$$

and the projection of this map to the second summand in the target is simply

$$
d \otimes 1: V_{2} \otimes V_{3} \rightarrow \Lambda^{2} V_{1} \otimes V_{3},
$$

which is injective since $d: V_{2} \rightarrow \Lambda^{2} V_{1}$ is injective by definition. Thus $H^{2}\left(\mathcal{M}_{3}\right)(5)=0$. Similarly

$$
H^{2}\left(\mathcal{M}_{3}\right)(6)=\operatorname{ker}\left(\Lambda^{2} V_{3} \rightarrow V_{1} \otimes V_{2} \otimes V_{3} \oplus \Lambda^{3} V_{2}\right)
$$

which vanishes because the projection of this map to the first summand in the target is the composition of the inclusion $\Lambda^{2} V_{3} \rightarrow V_{3} \otimes V_{3}$ of skew-symmetric 2-tensors, followed by the inclusion $V_{3} \otimes$ $V_{3} \rightarrow\left(V_{1} \otimes V_{2}\right) \otimes V_{3}$. 
To prove 5 , observe that

$$
V_{4}=\operatorname{ker}\left(V_{1} \otimes V_{3} \oplus \Lambda^{2} V_{2} \rightarrow \Lambda^{2} V_{1} \otimes V_{2}\right)
$$

If $\operatorname{dim}\left(V_{3}\right)=1$, then $V_{3} \subset V_{1} \otimes V_{2}$ has a basis element $b$ of the form

$$
b=\sum x_{i} \otimes c_{i}
$$

where the $c_{i}$ form a basis for $V_{2}$ and the $x_{i} \in V_{1}$. The map in question is then

$$
z=(v \otimes b, a) \rightarrow \sum\left(v \wedge x_{i}\right) \otimes c_{i}+a
$$

where $a \in \Lambda^{2} V_{2} \subset V_{2} \otimes V_{2}$ is a skew-symmetric tensor. If $z$ is in the kernel of this map, then

$$
\sum\left(v \wedge x_{i}\right) \otimes c_{i}=-a \in V_{2} \otimes V_{2}
$$

is a skew-symmetric tensor. But this is only possible if

$$
\sum\left(v \wedge x_{i}\right) \otimes c_{i}=0=a
$$

because for each $i, v \wedge x_{i}$ is decomposable, and $c_{i}$ is by assumption not decomposable. Thus $v \wedge x_{i}=0$ for all $i$. There are two possibilites: either $v=0$, in which case $C_{2}=V_{4}=0$, or $v \neq 0$, in which case there is only one nonzero $x_{i}$, call it $x$, and $v$ is a non-zero multiple of $x$, thus $C_{2}$ and $V_{4}$ are both one-dimensional with basis $x \otimes x \otimes c,(x \otimes x \otimes c, 0)$ respectively. Thus we see that $C_{2} \cong V_{4}$ as asserted.

\section{Corollary 0.2.}

1. $\mathcal{M}_{2}$ is 1-formal if and only if $V_{3}=0$.

2. $\mathcal{M}_{3}$ is l-formal if and only if $V_{4}=0$.

3. If $\operatorname{dim}\left(V_{3}\right)=1, \mathcal{M}_{3}$ is I-formal if and only if $C_{2}=0$.

From these assertions we see that the proofs of Example 2.6, Proposition 2.7 remain unchanged. In fact, the error affects only one assertion in the paper. Namely, the Lie algebra of Example 3.5 is not 3-step nilpotent, as asserted there. The reason is that the verification of assertion (3.2) of Lemma 3.4 is not sufficient for 3-step nilpotency (in this case $\operatorname{dim}\left(V_{3}\right)=5$, so part 5 of the lemma does not apply). One needs to compute the kernel of the map

$$
V_{1} \otimes V_{3} \oplus \Lambda^{2}\left(V_{2}\right) \rightarrow \Lambda^{2}\left(V_{1}\right) \otimes V_{2}
$$

which turns out to be one-dimensional. Using the notation $x_{1}, \ldots, x_{5}, c_{1}, c_{2}, c_{3}$ of Lemmas 3.3 and 3.4, a basis for the one-dimensional space $V_{4}$ is the following. Let $\alpha \in V_{1} \otimes V_{3} \subset V_{1} \otimes V_{1} \otimes V_{2}$ 
be

$$
\begin{aligned}
2 x_{4} & \otimes\left(x_{1} \otimes c_{1}+x_{2} \otimes c_{2}\right)+3 x_{2} \otimes\left(x_{4} \otimes c_{2}-x_{2} \otimes c_{3}\right) \\
& +x_{5} \otimes\left(x_{2} \otimes c_{1}+x_{5} \otimes c_{2}+x_{1} \otimes c_{3}\right)+x_{1} \otimes\left(x_{4} \otimes c_{1}+x_{3} \otimes c_{2}+x_{5} \otimes c_{3}\right),
\end{aligned}
$$

and let $\beta=c_{2} \wedge c_{1}=c_{2} \otimes c_{1}-c_{1} \otimes c_{2} \in \Lambda^{2} V_{2} \subset V_{2} \otimes V_{2}$. Then

$$
(\alpha, \beta) \in V_{1} \otimes V_{3} \oplus \Lambda^{2} V_{2}
$$

is a basis for $V_{4}$. We believe that further computation shows that the corresponding $\mathcal{M}_{4}$ is 1 -formal, but this would take us too far afield.

Second correction. The statement near the end of Section 5, "This is the subgroup of smallest index in the integral Heisenberg group which we know to be a Kähler group," is incorrect and misleading. The integral Heisenberg group itself is a Kähler group. This can be seen, for instance, by taking the first concrete example at the beginning of that paragraph, using a product of elliptic curves, with fundamental group the subgroup of the integral Heisenberg group (2.6) with $x_{1}, \ldots, x_{4}$ even. Its subgroup of index two where $z$ is also even is isomorphic to the integral Heisenberg group, and is of course a Kähler group since subgroups of finite index of Kähler groups are Kähler groups. This is a simple special case of the fact, pointed out to us by P. Deligne, that any two $\mathbb{Z}$-forms of the same nilpotent graded $\mathbb{Q}$-group can be embedded in each other as subgroups of finite index, therefore one is a Kähler group if and only if the other one is. 